\title{
Valoración económica de los impactos ambientales a la vegetación en la Concesión Forestal Carlos Edmundo Muñoz Landa, Marañón - Huánuco, 2021
}

Economic assessment of the environmental impacts on vegetation in the Carlos Edmundo Muñoz Landa Forest Concession, Marañón - Huánuco, 2021

Jairo Edson Gutiérrez Collao Universidad Nacional Agraria de la Selva jairo.gutierrez@unas.edu.pe

(iD) Luis Eduardo Oré Cierto Universidad Nacional Agraria de la Selva luisore21793@gmail.com

\section{RESUMEN}

Se evaluaron los impactos a la vegetación provocados por el aprovechamiento de recursos forestales maderables en la parcela de corta $\mathrm{N}^{\mathrm{o}} 05$ de la Concesión. Los impactos negativos se expresaron en valores monetarios. La investigación fue aplicada, transversal y descriptiva, en una muestra efectiva de 287 ha de una población de 8272 ha. Se aplicó el método inductivo, técnicas de línea base en las áreas asignadas para cada actividad del aprovechamiento forestal y observación directa de los impactos a la vegetación. Para la valoración económica de bienes se utilizó la Resolución Directoral Ejecutiva No 241-2016-SERFOR-DE (vegetación maderable) y Resolución Directoral Ejecutiva $\mathrm{N}^{\circ}$ 171-2016-SERFOR-DE (vegetación no maderable); mientras que para la valoración económica de servicios ecosistémicos se utilizó el valor de bono de carbono del año 2020 (24 euros = S/. 87,84). Se ocasionó un impacto negativo moderado (-553) por la destrucción de 1827 individuos maderables (66,64 m2 de área basal y 506,33 m3 de volumen) y 337 individuos no maderables, generando la pérdida de 99,66 t de carbono y apertura promedio de claros de 183,72 m2; que en valores monetarios se expresan en S/. 2 751,02 para bienes y S/. 8 753,86 para servicios (captura de carbono), haciendo un total de S/. 11 504,88.

Palabras clave: valoración económica, aprovechamiento de los recursos forestales maderables, concesión forestal, impactos ambientales. 


\begin{abstract}
Impacts to vegetation were evaluated provoked by exploitation of the timber forest resources on the $\mathrm{N}^{\circ} 05$ felling plot in the Concession. Negative impacts were expressed in monetary values. The research was applied, cross-sectional, and descriptive, with an effective sample of 287 acres from a population of 8272 acres. The inductive method was applied, with baseline techniques, to the areas assigned for each forest exploitation activity and the impacts on vegetation were observed directly. For the economic valuation of the economic goods the executive director's resolution $\mathrm{N}^{\circ}$ 241-2016-SERFOR-DE (timber vegetation) and the executive director's resolution $\mathrm{N}^{\circ}$ 171-2016-SERFOR-DE (non-timber vegetation) were used; meanwhile, for the economic valuation of the ecosystem services, the value of the carbon bond for the year 2020 was used (24 Euros $=$ S/. 87,84). There was a moderate negative impact $(-553)$ due to the destruction of 1827 timber specimens (base area of 66,64 $\mathrm{m} 2$ and volume of 506,33 m3) and 337 non-timber specimens, generating the loss of 99,66 tons of carbon and average clearings of clearings of 183,72 $\mathrm{m} 2$; which in monetary value is expressed as S/. 2 751,02 for goods and S/. 8 753,86 for services (carbon capture), creating a total of S/. 11 504,88 .
\end{abstract}

Keywords: economic valuation, utilization of timber forest resources, forest concession, environmental impacts

\section{INTRODUCCIÓN}

de bienes y servicios que tienen un valor económico, social y cultural para los seres humanos (Brüschweiler, Hoggel y Klay, 2004). Sin embargo, en los bosques húmedos tropicales se ha implementado el aprovechamiento convencional, cuyas características son: el carácter destructivo, el alto porcentaje de transformación de la cubierta vegetal original para convertirla a otros usos, la escasa integración bosque - industria, el empleo de maquinaria obsoleta y sub-utilizada, la baja utilización del potencial del bosque, la operación durante la temporada de menor precipitación, desperdicio excesivo y las malas condiciones de trabajo para los operarios (Orozco, 2006). Los impactos ambientales del aprovechamiento forestal convencional presentan en la actualidad ciertas características que alteran en forma negativa al ambiente y a los recursos de las áreas boscosas, provocando daños innecesarios al ecosistema y pérdidas económicas ocasionados por técnicas destructivas y planificadas inadecuadamente para la extracción de madera. Por tal motivo, se busca implementar el aprovechamiento de bajo impacto que está relacionado con la adecuación y ejecución de actividades extractivas planificadas previamente $\mathrm{y}$ sigilosamente, siendo controladas en el campo, 
reduciendo al mínimo la perturbación sobre el bosque residual y el suelo (Orozco, 2006). El aprovechamiento de impacto reducido, es un aprovechamiento sostenible, en el cual, la extracción de los productos forestales no debe exceder el nivel de regeneración durante el ciclo de corta, de esta manera se evita reducir irreversiblemente el potencial del bosque para producir madera comercializable (Maldonado, 2010). Por consecuencia, la puesta en marcha de las concesiones forestales en el Perú buscó el cambio de una actividad extractivista, raramente planificada; regularmente financiada por terceros y con escasa supervisión por el Estado hacia una actividad empresarial, donde la planificación, es vital y esencial; por ello, los concesionarios elaboran planes de manejo forestal para el cumplimiento de requisitos en las auditorías del Estado ya que contiene la información detallada de las actividades en campo a realizar (Colán et al., 2007).

Existe desconocimiento acerca del valor económico de los bienes y servicios ambientales, del grado de su agotamiento y deterioro, y sus efectos en el bienestar humano (Gorfinkiel, 1999). Entiéndase que el valor económico es la estimación en unidades monetarias del nivel de bienestar que un bien o servicio le genera a un individuo (MINAM, 2013). Así como también, puede ser la medición económica de beneficios ambientales perdidos, a lo que hay que sumarle servicios ambientales o ecosistémicos perdidos, ya sea para ejecutar las medidas en los estudios de impactos ambientales o para reparar los daños y pasivos ambientales; generados por el aprovechamiento de recursos naturales (Rebolledo, 2011). La valoración económica del patrimonio natural se ha ido implementado en la normativa ambiental como una herramienta útil y necesaria para la toma de decisiones informadas con respecto al aprovechamiento sostenible de los recursos naturales (MINAM, 2015).

Por otro lado, Sención (2002), en su investigación confirmó que los servicios ecosistémicos generan mayor valor económico que los bienes. Además, indica que es vital contar con datos ecológicos de producción del bosque, precios económicos de bienes/sustitutos y costos económicos de transformación y comercialización para realizar una valoración económica adecuada. Aparte, Llerena, Malleux y Chang (1980) en una investigación concluyó que los bosques se hallan en condición de mucha extracción, sobretodo, de especies altamente valiosas como el ishpingo y la caoba, que conlleva a una devaluación en relación a su estado original. También, determinó que los bosques de tipo 1 y 2 con un total de 5446 ha presentan un valor promedio de S/. 82 484/ha, para el año 1978. Por lo tanto, la valoración económica es una herramienta indispensable para la evaluación de impactos al ambiente y para la ejecución de políticas ambientales (Casiano, 2015).

La valoración económica de los impactos a la vegetación maderable y no maderable (bienes y servicios) provocados por el aprovechamiento de los recursos forestales maderables en la parcela de corta $\mathrm{N}^{\circ} 05$ de la concesión forestal Carlos Edmundo Muñoz Landa, está en función a la planificación previa, a la técnica de aprovechamiento implementado, al número de individuos impactados, al valor al estado natural por categorías de las especies maderables, a la forma de venta y precios del mercado de las especies no maderables y al precio de 
la tonelada de carbono en el mercado mundial. En esta investigación se presenta el cálculo del valor económico del impacto a la vegetación (maderable y no maderable) provocados por el aprovechamiento de 20 árboles comerciales aprovechables.

\section{METODOLOGÍA}

\section{Área de estudio}

Esta investigación fue realizada en la parcela de corta $\mathrm{N}^{\mathrm{o}} 05$ de la concesión forestal Carlos Edmundo Muñoz Landa, distrito La Morada, provincia Marañón, región Huánuco. El área efectiva de estudio comprende una extensión de 287 ha.

\section{Metodología para evaluar la vegetación}

Las actividades del aprovechamiento realizadas en la parcela de corta $\mathrm{N}^{\mathrm{o}} 05$ fueron: instalación de campamento, apertura de caminos (primarios, secundarios y entradas), apertura de patios de troza, tumba y trozado de 20 árboles comerciales y arrastre de las trozas.

En las áreas asignadas para el campamento, patios de acopio y tumbado - trozado de los 20 árboles comerciales, así como en los trazos realizados para la apertura de caminos y arrastre se realizó el inventario al $100 \%$ de la vegetación maderable (árboles con diámetro a la altura del pecho mayor a $10 \mathrm{~cm}$ ), en $10 \mathrm{~s}$ que, se determinó el diámetro a la altura del pecho (cm), altura comercial y total (m). Mientras que en la vegetación no maderable (palmeras, ornamentales, etc.) se determinó el número de hojas y la altura total.
Para finalizar, con la ayuda de un especialista forestal en dendrología, se identificaron los nombres comunes y nombres científicos de la vegetación. Se realizaron dos inventarios, uno antes del aprovechamiento y otro después del aprovechamiento.

Exclusivamente para la evaluación del tumbado de árboles comerciales se instalaron seis cuadrantes de 40 ha cada una. En cada cuadrante se instalaron en promedio cuatro parcelas redondas de 0,38 ha cada una. En total se instalaron 20 parcelas redondas para evaluar 20 árboles comerciales. Las parcelas redondas se seccionaron en cuatro cuadrantes orientados con azimut de $0^{\circ}, 90^{\circ}, 180^{\circ}$ y $270^{\circ}$.

\section{Análisis de datos}

Aparte de determinar el número de individuos y el número de especies, con el diámetro a la altura del pecho se obtuvo el área basal de cada individuo. Asimismo, con el área basal obtenida y con la altura comercial de los individuos se procedió a determinar el volumen comercial.

Se compararon los datos obtenidos al inicio y al final, obteniéndose de esta manera el impacto (pérdida de individuos) para individuos maderables y no maderables.

En todas las parcelas donde se realizaron las actividades se calcularon la biomasa aérea viva (BAV). Para ello se utilizó la fórmula propuesta por Cháve et al., (2005) que es utilizada para las especies de bosques húmedos tropicales que consideran el diámetro y densidad de madera como las únicas variables predictoras.

Para obtener las densidades de las especies maderables impactadas durante el aprovechamiento 
forestal se utilizó el Database Tree Functional Atributes and Ecological "Wood Density".

Para calcular la biomasa aérea de las palmeras impactadas se utilizó la ecuación publicada por Pearson et al., (2005) que considera la altura total como única variable predictora.

Para las estimaciones de las fijaciones o capturas de carbono se asume que el contenido de carbono corresponde al 50\% de la biomasa aérea de los árboles vivos (Chave et al., 2005). La fórmula utilizada en la investigación, fue la siguiente:

Se realizó la comparación entre la vegetación original y la vegetación residual (post aprovechamiento). Para determinar el valor económico nos guiamos en la Resolución Directoral Ejecutiva $\mathrm{N}^{\circ}$ 241-2016SERFOR-DE.

Por otro lado, la vegetación no maderable fue evaluada de acuerdo a la homogeneización por medidas realizadas, por unidad de medida (kilogramos, número de hojas, etc.). Se realizó la comparación entre la vegetación original y la vegetación residual (post aprovechamiento). Se identificaron los precios o valores de los productos no maderables. Utilizando los valores considerados en la lista de la Resolución Suprema N 010-2003-AG con los valores considerados en la Resolución Directoral Ejecutiva $\mathrm{N}^{\circ}$ 171-2016-SERFOR-DE. Primero se determinó la tasa de variación o crecimiento,

Para estimar el valor del producto al estado natural correspondiente al año de interés (2020) se utilizó la tasa de variación determinada en la regresión lineal y como base, los valores al estado natural de los productos forestales diferentes a la madera.

Asimismo, se determinó en términos monetarios la pérdida del servicio ecosistémico "captura de carbono", para ello, se investigó el precio que se paga en el mercado actualmente por una tonelada de $\mathrm{CO}_{2}$ "bono de carbono", el cual es de 24 euros $(87,84$ soles).

\section{Variables de la investigación}

Variable X: Valoración económica de los impactos ambientales.

\section{Indicadores:}

X1 : Variabilidad del valor al estado natural (S/. $/ \mathrm{m}^{3} \mathrm{r}$ ) por categoría de las especies maderables.

X2 : Variabilidad del valor al estado natural (S/.) por formas de venta de las especies no maderables.

X3 : Variabilidad del valor económico de captura de carbono (S/.) por cantidad de carbono almacenado según especie maderable y no maderable.

X4 : Variabilidad de la multa (S/. o U.I.T) de acuerdo al perjuicio económico, gravedad de daños, afectación a la conservación de la especie de flora según categoría de amenaza y a la afectación a la función en la regeneración de la especie.

Variable Y: Vegetación en la Concesión Forestal Carlos Edmundo Muñoz Landa.

\section{Indicadores:}

Y11: Número de individuos maderables y no maderables impactados provocados por la 
implementación de actividades del aprovechamiento de los recursos forestales maderables.

\section{RESULTADOS}

\section{Cálculo del valor económico de los impactos a la vegetación (maderable y no maderable) por el aprovechamiento de 20 árboles comerciales}

La Tabla 1 muestra el valor económico de los impactos a la vegetación provocado por las actividades del aprovechamiento de 20 árboles comerciales. El 94,05\% del valor económico del impacto corresponde a la pérdida de individuos maderables.

El 57,3\% del valor económico del impacto concierne a la "apertura de caminos" (S/. 1.575,98). El 29,3\% incumbe a la "apertura de patios de acopio" (S/. $807,24)$. El 10\% se atribuye al "tumbado de árboles seleccionados" (S/. 276,07). El 2,3\% corresponde a la "instalación de campamento" $(\mathrm{S} / .63,16)$ y $1 \%$ para el "arrastre de trozas de los árboles seleccionados" (S/. 28,58).

\section{Tabla 1}

Valor económico de impactos negativos a la vegetación

\begin{tabular}{lccc}
\hline \multirow{2}{*}{ Tipo de Vegetación } & $\begin{array}{c}\text { Valor Económico Inicial } \\
(\mathrm{S} / .)\end{array}$ & $\begin{array}{c}\text { Valor Económico Final } \\
(\mathrm{S} / .)\end{array}$ & $\begin{array}{c}\text { Valor Económico del } \\
\text { Impacto }(\mathrm{S} / .)\end{array}$ \\
\hline Maderable & 7308,92 & 4721,70 & 2587,22 \\
No Maderable & 279,75 & 115,94 & 163,80 \\
\hline \multicolumn{1}{c}{ Total } & 7588,67 & 4837,64 & 2751,02 \\
\hline
\end{tabular}

El valor económico está agrupado por categorías de especies con sus respectivos valores al estado natural por $\mathrm{m} 3$ rollizo. El $56,1 \%$ del volumen comercial

\section{Tabla 2}

Valor económico de vegetación maderable según categoría

\begin{tabular}{cccc}
\hline Categoría & Valor $(\mathrm{S} /)$. & $\mathrm{N}^{\mathbf{o}}$ Individuos & Porcentaje \\
\hline B & 12,00 & 278 & 15,22 \\
C & 6,00 & 164 & 8,98 \\
D & 4,00 & 360 & 19,70 \\
E & 2,00 & 1.025 & 56,10 \\
\hline
\end{tabular}

Del mismo modo, la Tabla 4 especifica que los valores económicos para vegetación no maderable impactada, se agruparon por especies y forma de venta.

El 44,3\% del valor económico total corresponde a Chamaerops humilis L., "palmiche". El 21,8\% corresponde a especies de valor económico futuro "E” (Tabla 3). 
compete a Heliconia rostrata Ruíz \& Pav., "pico de loro" (se vende en el mercado por docena de hojas).
El $0,5 \%$ atañe a Geonoma poeppigiana Martius "palmichillo".

Tabla 4

Valor económico de la vegetación no maderable según forma de venta

\begin{tabular}{|c|c|c|c|c|}
\hline Nombre Científico & $\begin{array}{l}\text { Forma de } \\
\text { Venta }\end{array}$ & Cantidad & $\begin{array}{c}\text { Valor (S/.) - } \\
2020\end{array}$ & $\begin{array}{c}\text { Valor Económico } \\
\text { Total (S/.) }\end{array}$ \\
\hline Calathea luthea A. & Decena & 9,4 & 2,04 & 19,18 \\
\hline Carludovica palmata Ruíz et Pavón & $\mathrm{Kg}$ & 88,1 & 0,138 & 12,16 \\
\hline Chamaedorea pinnatifrons (Jacq.) & $\mathrm{Kg}$ & 258,5 & 0,138 & 35,67 \\
\hline Chamaerops humilis L. & $\mathrm{Kg}$ & 525,9 & 0,138 & 72,57 \\
\hline Geonoma poeppigiana Martius & $\mathrm{Kg}$ & 6,2 & 0,138 & 0,86 \\
\hline Heliconia rostrata Ruiz \& Pav. & Docena & 0,7 & 10,71 & 7,14 \\
\hline Socratea exorrhiza (Mart.) H. Wendl. & $\mathrm{Kg}$ & 117,6 & 0,138 & 16,23 \\
\hline & & & & 163,80 \\
\hline
\end{tabular}

Valor económico por pérdida de servicios ecosistémicos

La Tabla 5 explica y muestra el valor económico total de la pérdida del servicio ecosistémico "captura de carbono" provocados por las diferentes actividades del aprovechamiento de los recursos forestales maderables.

El valor económico total por pérdida del mencionado servicio ecosistémico se obtuvo de acuerdo al precio de mercado de una tonelada de carbono. La cantidad de carbono perdido por impactos a la vegetación maderable, supera en 97,6\% a la cantidad de carbono perdido por impactos a la vegetación no maderable.

Del total del valor económico del carbono perdido por impactos a la vegetación maderable, el 57,3\% corresponde a la actividad "apertura de caminos", secundado por la actividad "apertura de patios de acopio" con $28,5 \%$ y seguido por la actividad “tumbado de árboles" con 11,6\%.

Al comparar el valor económico de pérdida de carbono de los tipos de vegetación impactados, el valor económico de la vegetación maderable es $97,6 \%$ mayor que el valor de la vegetación no maderable.

\section{Tabla 5}

Valor económico de la pérdida de captura de carbono

\begin{tabular}{lccc}
\hline \multirow{2}{*}{ Actividad } & \multicolumn{2}{c}{ Carbono Perdido (t) } & Valor Económico del \\
& Impacto (S/.) & Vegetación No \\
Maderable & Vegetación Maderable & 0,11 & 138,60 \\
Instalación de Campamento & 1,47 & 1,24 & 5011,98 \\
Apertura de Caminos & 55,82 & 0,53 & 2491,52 \\
Apertura de Patios de Acopio & 27,83 & 0,45 & 1015,62 \\
Tumbado & 11,12 & 0,00 & 96,13 \\
Arrastre & 1,09 & 2,33 & 8753,86 \\
\hline \multicolumn{2}{c}{ Total } & 97,33 & \\
\hline
\end{tabular}




\section{Contrastación de hipótesis de investigación}

H1. La valoración económica de impactos a la vegetación provocada por el aprovechamiento de 20 árboles comerciales en la parcela de corta $\mathrm{N}^{\circ} 05$, es alta, debido a la inadecuada planificación e implementación del aprovechamiento de los recursos forestales maderables empleado.

H0. La valoración económica de impactos a la vegetación provocada por el aprovechamiento de 20 árboles comerciales en la parcela de corta $\mathrm{N}^{\circ} 05$, es baja, debido a la inadecuada planificación e implementación del aprovechamiento de los recursos forestales maderables empleado
Al considerar la pérdida de bienes, servicio e incluida la multa por daños al patrimonio forestal, el valor económico impactado representa el 35,5\% del valor económico inicial del área antes del aprovechamiento, por consecuencia, rechazamos la hipótesis alternativa (H1) y aceptamos la hipótesis nula (H0). A pesar que la investigación es descriptiva, se procedió a determinar la correlación de variables dependientes e independientes. En primer lugar se verificó la normalidad de los datos utilizando la prueba de Shapiro - Wilk. Como el $\mathrm{P}$ valor es > a 0,05 , concluimos que los tres indicadores de la variable dependiente presentan datos con distribución normal (Tabla 6).

\section{Tabla 6}

Prueba de normalidad Shapiro - Wilk de la variable dependiente

\begin{tabular}{|c|c|c|c|}
\hline Indicadores & Estadístico & $\mathrm{gl}$ & Sig. \\
\hline $\mathrm{N}^{\mathrm{o}}$ de Individuos Impactados & 0,822 & 6 & 0,091 \\
\hline Valor Económico Natural de Individuos Impactados & 0,800 & 6 & 0,059 \\
\hline Valor Económico por Pérdida de Carbono & 0,804 & 6 & 0,064 \\
\hline
\end{tabular}

En la Tabla 7, se observa la correlación entre los indicadores "valor económico natural de individuos impactados" (maderable y no maderable) y "valor económico por pérdida de carbono" con el indicador número de individuos impactados en cada actividad del aprovechamiento forestal.

\section{Tabla 7}

\section{Coeficiente de correlación de Pearson}

\begin{tabular}{clcc}
\hline \multicolumn{2}{c}{ Características } & Valor Económico Natural de & Valor Económico por Pérdida de \\
Individuos Impactados & Carbono
\end{tabular}

Al ser el $\mathrm{P}$ valor $<$ a 0,05 , se concluye que existe correlación y sumado a ello la correlación de Pearson de 0,981 y 0,987 indican una alta correlación entre los indicadores.

** La correlación es significativa en el nivel 0.01 (2 colas). 


\section{DISCUSIÓN}

En lo que concierne a valores económicos, Llerena, Malleux y Chang (1980), en una investigación mediante un método empleado para evaluar y valorizar un bosque tropical sometido a intensa y constante explotación, determinó que los bosques de tipo 1 y 2 con un total de 5446 hectáreas presentan un valor promedio de S/. 82 484,00 por hectárea, para el año 1978. En general, los bosques se hallan en una condición de intervención o fuerte entresaque, principalmente de especies valiosas como el ishpingo y caoba, por lo cual, se ha desvalorizado en relación a su estado original. Con estos resultados no se puede discutir porque en la investigación no se valoró el bosque (parcela de corta $\mathrm{N}^{\circ} 05$ ), sino que se valoraron los impactos a la vegetación (maderable y no maderable).

El valor económico calculado en la investigación no fue producto del bienestar a un individuo generado por un bien o servicio, sino, fue necesaria para la toma de decisiones por parte del regente forestal y del concesionario con respecto a los valores económicos por pérdidas de bienes y servicios, incluida la multa por daños al patrimonio forestal; es por ello, que está más relacionado con lo aseverado por IIAP (2009), quien asegura que, la valoración económica de los recursos tiene su base teórica en el análisis económico, en la teoría del bienestar y cambios en el bienestar social, a lo que hay que sumarle el bienestar ambiental. Del mismo modo, los resultados de la investigación van a permitir, tal como lo sugiere Casiano (2015), la ejecución de políticas ambientales. En consecuencia, se confirma lo mencionado por Rebolledo (2011), quien señala que, la valoración económica de impactos es la medición económica de beneficios ambientales perdidos, a lo que hay que sumarle servicios ambientales o ecosistémicos perdidos, ya sea para ejecutar las medidas en los estudios de impactos ambientales o para reparar los daños y pasivos ambientales; generados por el aprovechamiento de recursos naturales.

En otra parte, la investigación corrobora que los bosques son muchos más valiosos por sus servicios ecosistémicos que por su producción de bienes; por lo tanto, los servicios ecosistémicos son insustituibles, debido a que son esenciales para la vida. Por ende, tal como indica Ocampo (2017), la preservación de los bosques naturales debe ser una prioridad para la población por su papel en la provisión de agua, reducción de emisiones de gases de efecto invernadero y conservación de la biodiversidad.

\section{CONCLUSIONES}

Se calculó que el valor económico total por impacto a la vegetación maderable y no maderable fue de S/. 2 751,02 en bienes y de S/. 8.753,86 en servicios ecosistémicos, haciendo un total de S/. 11 504,88, a razón de S/. 5,32 por individuo impactado (muerto). El valor económico por la extracción de un árbol comercial, expresado en bienes y servicios, es de $\mathrm{S} /$. 575,24; si estimamos al aprovechamiento de 1415 árboles comerciales de S/. 813 970,26. Dicho dato estimado estaría sesgado porque no se tiene certeza del impacto que generaría el tumbado de 1415 árboles. Haciendo una inferencia de acuerdo a los porcentajes de impactos a la vegetación remanente, al porcentaje del valor económico de las especies según su categoría y su forma de venta, el valor económico 
estimado de los impactos a la vegetación (bienes y servicios) provocados por el aprovechamiento de 1 415 árboles comerciales, sería S/. 384 817,39.

\section{REFERENCIAS}

[1] Brüschweiler, S., Hoggel, U. \& Klay, A. (2004). Los bosques y el água: Interrelaciones y su manejo. Berna.

[2] Casiano, C. (2015). Valoración económica del impacto en los servicios ecosistémicos del bosque de ribera en cabecera de cuenca del río Utcubamba, distrito de Leimebamba, provincia de Chachapoyas, región Amazonas, Perú; 2014 -2015 . Chachapoyas.

[3] Chave, J., Andalo, C., Brown, S., Cairns, M., Chambers, J., Eamus, D., Fôlster, H., Fromard, F., Higuchi, N., Kira, T., Lescure, J., Nelson, B., Ogawa, H., Puig, H., Riéra, B. y Yamakura, T. (2005). Alometría de árboles y mejor estimación de las reservas de carbono y el equilibrio en los bosques tropicales. Oecología 145:87-99.

[4] Colán, V., Sabogal, C. y Catpo, J. (2007). Monitoreo de operaciones de manejo forestal en concesiones con fines maderables de la Amazonía peruana. Manual de campo para la evaluación del impacto de las operaciones de aprovechamiento en concesiones forestales con fines maderables en la Amazonía peruana. Pucallpa.

[5] Gorfinkiel, D. (1999). La valoración económica de los bienes ambientales: una aproximación desde la teoría y la práctica.
[6] IIAP (2009). Valoración económica de bienes y servicios en ecosistemas de bosques inundables y de altura de la Amazonía peruana: Marco conceptual y propuesta metodológica. Iquitos.

[7] Llerena, C., Malleux, J. y Chang, A. (1980). Evaluación y valorización de un bosque tropical en explotación. Lima.

[8] Maldonado, H. (2010). Evaluación de la sostenibilidad del manejo forestal implementado en los resguardos indígenas de Urabá, Antioquía, Colombia. Antioquía.

[9] MINAM (2013). Guía de valoración económica de impactos ambientales. Resolución Ministerial N³87-2013-MINAM. Lima.

[10] MINAM (2015). Guía nacional de valoración económica del patrimonio natural. Lima.

[11] Ocampo, K. (2017). Retos asociados al uso del suelo: El dilema entre conservación y producción en los Bosques Andinos de Robles del Corredor Ecológico Guantiva - La Rusia Iguaque.

[12] Orozco, L., Brumér, C. y Quirós, D. (2006). Aprovechamiento de impacto reducido en bosques latifoliados húmedos tropicales. Turrialba.

[13] Pearson, T., Walker, S. y Brown, S. (2005). Libro de consulta sobre uso de la tierra, cambio de uso de la tierra y proyectos forestales.

[14] Rebolledo, D. (2011). Manual para la valoración social de: impactos y daños Ambientales de actividades agrícolas. Caracas. 
[15] Sención, G. (2002). Valoración económica de un ecosistema: Bosque tropical Petén, Guatemala.

[16] SERFOR (2016). Lineamientos para la elaboración del Plan General de Manejo Forestal para concesiones forestales con fines maderables. Resolución de Dirección Ejecutiva $N^{\circ} 046$ - 2016 - SERFOR - DE. Lima, Perú.

[17] SERFOR (2016). Metodología para la determinación del valor al estado natural de productos forestales diferentes a la madera para el pago del derecho de aprovechamiento. Resolución de Dirección Ejecutiva $N^{\circ} 171$ 2016 - SERFOR - DE. Lima, Perú.

[18] SERFOR (2016). Metodología para la determinación del valor al estado natural de la madera para el pago de derecho del aprovechamiento y los valores al estado natural de la madera. Resolución de Dirección Ejecutiva $N^{\circ} 241$ - 2016 - SERFOR - DE. Lima, Perú.

[19] SERFOR (2018). Lineamientos para la aplicación de los criterios de gradualidad para la imposición de la sanción pecuniaria. Resolución de Dirección Ejecutiva $\mathrm{N}^{\circ} 004-$ 2018 - SERFOR - DE. Lima, Perú. 